\title{
Cooperativity between water and lipids in lamellar to inverted-hexagonal phase transition
}

\author{
Mafumi Hishida $^{1 *}$, Koichiro Tanaka $^{2,3}$, Yasuhisa Yamamura ${ }^{1}$ and Kazuya Saito ${ }^{1}$ \\ ${ }^{1}$ Department of Chemistry, Faculty of Pure and Applied Sciences, University of Tsukuba, \\ Tsukuba, Ibaraki 305-8571, Japan \\ ${ }^{2}$ Institute for Integrated Cell-Material Sciences, Kyoto University, Kyoto 606-8501, Japan \\ ${ }^{3}$ Core Research for Evolutional Science and Technology (CREST), Japan Science and \\ Technology Corporation (JST), Japan
}

It has been unclear whether the role of water in the self-assembly of soft materials and biomolecules is influential or water is just a background medium. Here we investigate the correlation between hydration state of lipid membrane and structural phase transition of the membrane between lamellar and inverted-hexagonal phases, as an intermediate process of membrane fusion, by using the complementary techniques of X-ray scattering and terahertz (THz) spectroscopy. By comparing two lipid species, our results indicate that the structural changes of the lipid membrane depend on the behavior of the surrounding water, especially in the second hydration layer, in addition to the molecular shape of lipids. The water behaves differently at each membrane surface owing to the different hydrophilicities of the lipid head groups.

KEYWORDS: lipid membrane, hydration water, lamellar phase, inverted-hexagonal phase, membrane fusion, $\mathrm{THz}$ spectroscopy, small-angle $\mathrm{x}$-ray scattering, grazing-incidence small-angle $\mathrm{x}$-ray scattering

\section{Introduction}

Water is essential for all forms of life, but its exact role in biological systems is still unclear. A key component to unravelling this mystery is to understand the importance of the molecular interaction between water and biological molecules. Most biomolecules exhibit their respective functions after self-assembling into various conformations, but the role of water in this process is not fully understood. ${ }^{1,2)}$ The molecular interactions are reflected in the hydration states of the biomolecules, and a correlation between the self-assembly mechanism and the molecular hydration state would shed light on the essential nature of water not only

${ }^{*}$ E-mail: hishida@chem.tsukuba.ac.jp 
in biological systems but also in many soft materials dissolved in water such as polymers, surfactants, and colloids.

Self-assembly has been widely analyzed and interpreted based on a balance between attractive and repulsive interactions as typified by DLVO theory, ${ }^{3-5)}$ in which water is assumed to be a homogeneous medium and the hydration state of the solute at the molecular level is entirely ignored. Dynamical structures of the hydration state that changes gradually from the solute surface have been considered previously:5) The layer where water is strongly trapped by the solute is idiomatically called as the "first hydration shell", and the layer where the water dynamics are slightly perturbed is called as the "second hydration layer", even although a clear boundary between these layers does not exist supposedly. However, there is no commonly accepted definition of hydration water and even the exact amount of hydration water is still a matter of dispute. Infrared (IR) spectroscopy, nuclear magnetic resonance (NMR), and neutron scattering have revealed the dynamical properties of the first hydration shell, ${ }^{6-8)}$ but the second hydration layer remains essentially unknown. The behavior of water in the second hydration layer, however, is likely to be correlated with the self-assembly of biomolecules, since the thickness of the second hydration layer is comparable to the length scale of various mesoscale interactions, such as the van der Waals interaction, between self-assembled structures.

Since water molecules in the bulk phase have reorientational dynamics on a time scale of $10^{-12}$ to $10^{-13} \mathrm{~s},{ }^{9,10)}$ this is the ultrafast time scale on which the dynamics must be observed to precisely detect the hydration state and second hydration layer. Computer simulations can be employed to this end, and recently, some molecular dynamics (MD) simulations have indicated that the hydration at the protein surface is long range (on a nanometre scale), ${ }^{11)}$ has significantly different properties from bulk water, ${ }^{12)}$ and dominates the self-assembly process of a protein. ${ }^{13)}$ However, the lack of a suitable experimental method for observing the water dynamics means that the correlation between the hydration state and the biomolecular selfassembly cannot be verified.

Recent progress in the field of ultrafast laser technology, allowing spectroscopy in the $\mathrm{THz}$ $\left(10^{12} \mathrm{~Hz}\right)$ frequency range, offers a solution to this dilemma. ${ }^{15,16)}$ We previously applied this technique to biomolecular systems, i.e., lipid bilayer membranes, the basic building blocks of biomembranes, ${ }^{17)}$ and in combination with X-ray scattering to observe the stacking structure, we were able to clarify the precise hydration state of a lipid membrane and revealed that the overall hydration layer has a longer range than the previously observed first hydration shell, extending up to $1 \mathrm{~nm}$ from the surface. This result confirmed that $\mathrm{THz}$ spectroscopy gives the 
information of overall hydration state with including the slightly perturbed hydration water, in addition to the previously observed first hydration shell. Thus, in the present study, we define that the slightly perturbed water observed by THz spectroscopy is water in the second hydration layer. The length scale of the overall hydration state is comparable to that of the van der Waals interaction and the electrostatic interaction between membranes, ${ }^{18)}$ which implies that a strong correlation would be expected among the hydration state, the intermembrane interaction, and the self-assembly structures. A later study using a surfactant $\left(\mathrm{C}_{12} \mathrm{E}_{5}\right)$ confirmed this prediction: ${ }^{19)}$ the hydration state of the surfactant exhibits transition-like behavior that coincides with the structural phase transitions from the hexagonal to micellar to lamellar phases. Such a correlation is also observed at the gel-to-liquid-crystalline phase transition of a phospholipid membrane. ${ }^{20)}$

Membrane fusion of a lipid bilayer is a basic biomembrane function accompanied by a structural change of the membrane ${ }^{21)}$ that arises universally in a cell during endocytosis, exocytosis, and cell division. From a soft matter physics view point, the membrane fusion is considered to proceed as a result of lamellar to inverted-hexagonal phase transition, ${ }^{24,25)}$ although the molecular mechanism of the membrane fusion is not fully understood. Since negative curvature of the lipid monolayer is favorable to achieve this transition, ${ }^{5,23)}$ a lipid molecule with a small head group easily undergoes this transition. Actually, it is known that decreasing water content between membranes leads to form non-lamellar phases, ${ }^{26)}$ and lipid molecules with a phosphatidylethanolamine (PE) head group are known to easily undergo the transition from lamellar to inverted-hexagonal phases, compared to the lipids with phosphatidylcholine (PC) head groups (the molecular structure is shown in Fig. 1), even though PC lipid exhibits the transition under particular circumstances when the hydrophobic acyl chains have large volume and the membrane has negative curvature. ${ }^{27,28)}$ Actually in a living system, PE lipid is known to be necessary for the membrane fusion. ${ }^{22)}$ However, for the phase transition from lamellar to inverted-hexagonal phases to be successful, the two membranes have to be partially dehydrated and close each other. It is then plausible that water works cooperatively with the membrane structural changes, as predicted by MD simulations. ${ }^{29)}$

In the present study, we investigated this cooperativity between the long-range hydration state and the phase transition between lamellar and inverted-hexagonal phases of the lipid membranes through a combination of $\mathrm{THz}$ spectroscopy and X-ray scattering techniques. The membranes considered here consisted of synthesized PE and PC lipids (see Methods); the head groups only differ by the presence of choline (Fig. 1), and three methyl groups at the quaternary ammonium group of the PC lipid are replaced by hydrogen atoms to yield the PE 
lipid (primary ammonium group). Lamellar structures of the lipid membranes are observed using small-angle X-ray scattering (SAXS), and the long-range hydration states of each lipid membrane are observed by attenuated total reflection (ATR) THz time-domain spectroscopy (THz-TDS) ${ }^{30)}$ The structural change of the membranes from the lamellar to inverted hexagonal phase was investigated using grazing-incidence small-angle X-ray scattering (GISAXS) ${ }^{24)}$ by controlling the hydration states of the lipid membranes through changes in the atmospheric humidity. This multifaceted approach was essential in revealing the hydration dependence of the self-assembly in detail.

\section{Materials and methods}

\subsection{Sample preparation}

1-Palmitoyl-2-oleoyl-sn-glycero-3-phosphocholine and 1-palmitoyl-2-oleoyl-sn-glycero3-phosphoethanolamine (POPC and POPE, respectively) were obtained from Avanti Polar Lipids, Inc. (Alabaster, Alabama, USA), in powder form and used without further purification. Both these lipids are neutral phospholipids without counter ions, which is crucial for the terahertz time-domain spectroscopy (THz-TDS) measurements, since the hydration state of the counter ions is also detected in addition to that of the lipid membranes. For the small-angle X-ray scattering (SAXS) and THz-TDS measurements, the powder samples were dissolved in pure water (MilliQ) to produce solutions with different molar ratios of $R=\left[\mathrm{H}_{2} \mathrm{O}\right] /[$ lipid $]=95,125$, and 155. The lipids formed multilamellar vesicles. For the grazing-incidence small-angle X-ray scattering (GISAXS) measurements, the POPC and POPE powders were dissolved in a mixture of chloroform and methanol (2:1 v/v) to yield a solution of $10 \mathrm{mM}$, and $3 \mu \mathrm{L}$ of the lipid-organic solvent solution was deposited on a silicon wafer surface $(10 \mathrm{~mm} \times 10 \mathrm{~mm})$. After the organic solvents had evaporated, a dried phospholipid film remained on the wafer. All the SAXS, THz-TDS, and GISAXS measurements were performed at a constant temperature of $50{ }^{\circ} \mathrm{C}$; both POPC and POPE are known to be in the liquid crystalline $\left(\mathrm{L}_{\alpha}\right)$ phase at this temperature. To measure the $\mathrm{THz}$ spectrum of partially dried lipid films, the dried films $2-3 \mathrm{~mm}$ in thickness were also made on a sample cell by evaporating the organic solvent as performed for GISAXS; these films were much thicker than the penetration depth of the evanescent field of the $\mathrm{THz}$ wave in our attenuated total reflection (ATR) cell. The THz-TDS measurements on the partially dried lipid films were performed at relative humidity $(\mathrm{RH})$ of $65 \pm 5 \%$. Ammonium chloride and tetramethylammonium chloride were obtained from Nacalai Tesque, Inc. (Kyoto, Japan), in powder form and used without further purification. The powders were dissolved in pure water (MilliQ) to 
yield a $2 \mathrm{M}$ solution. The THz-TDS measurements were performed at $25^{\circ} \mathrm{C}$.

\subsection{Small-angle $X$-ray scattering}

SAXS measurements were performed at the BL-15A and BL-6A beamlines of the Photon Factory, High Energy Accelerator Research Organization, Japan. X-rays with a $1.5 \AA$ wavelength were used, and the rays were detected using a charge-coupled device (CCD; C7300, Hamamatsu Photonics (Hamamatsu, Japan)) with an image intensifier. The sampleto-detector distance was about $1 \mathrm{~m}$, calibrated using standard samples (lead stearate and silver behenate). The temperature of the sample solutions was controlled by using a hot stage (FP900 thermo system with a FP82HT hot stage, Mettler Toledo (Greifensee, Switzerland)). From a sharp Bragg peak at $q_{0}$ due to regular stacking of the lipid membranes in the multilamellar vesicles, the lamellar repeat distance was calculated according to $d=2 \pi / q_{0}$. Since the membrane thicknesses $d_{b}$ are known to be $37.9 \AA$ for POPC ${ }^{31)}$ and $38.5 \AA$ for POPE, ${ }^{32)}$ the water layer thicknesses $d_{w}$ were obtained by subtracting the membrane thicknesses from the lamellar repeat distances, $d_{w}=d-d_{b}$.

\subsection{Terahertz time-domain spectroscopy}

THz-TDS measurements were performed using the setup reported previously. ${ }^{17,19,30,34)}$ An Er-doped fibre laser (Femtolite, IMRA America, Inc. (Ann Arbor, Michigan, USA); 780 $\mathrm{nm}, 100 \mathrm{fs}, 50 \mathrm{MHz}$ ) was used as a light source. The $\mathrm{THz}$ waves were generated using a dipole photoconductive antenna and detected by an electro-optic sampling method using a (1 10 )-oriented ZnTe crystal. A Dove prism made of silicon (refractive index of 3.4) was used as the sample cell; this enabled an ATR measurement for an accurate determination of the complex dielectric function of the aqueous solutions. The incident angle of the THz wave was $\theta=51.6^{\circ}$, and the prism was arranged so as to obtain a $p$-polarization geometry to detect even a small change in the dielectric constant induced by the hydration effect of the solute. The penetration depth of the evanescent field of the THz wave was about $20 \mu \mathrm{m}$. The temperature of the ATR sample cell was controlled using a Peltier device with PID control. For the TDS results, the Kramers-Kronig relation is not required to obtain the complex dielectric constant. As a result, both the real and imaginary parts of the complex dielectric constants could be precisely determined with high reliability in the $0.3-2.7 \mathrm{THz}$ region in our experiments.

\subsection{Grazing-incidence small-angle $X$-ray scattering}

GISAXS measurements were performed at the BL23A beamline of the National Synchrotron Radiation Research Center, Taiwan. X-rays of a $0.82 \AA$ wavelength were used, the 
sample-to-detector distance was about $1.8 \mathrm{~m}$, and rays were detected using a CCD (MAR165, Marreseach, Norderstedt, Germany). The grazing-incidence angle was kept constant at $0.2^{\circ}$. The lipid samples on the silicon wafers were placed in a self-made temperature-humidity chamber with windows for the X-rays; the temperature was controlled independently at the sample stage, water bath, and jacket for the whole chamber. Specular reflection of the X-rays was attenuated using two beam stoppers. The GISAXS measurements were performed with respect to each about $5 \%$ of relative humidity. For each change of $5 \%$ relative humidity, the samples were rested for at least $5 \mathrm{~min}$ and equilibration of the lamellar structures were confirmed. Measurements were performed on both processes of increasing and decreasing humidity to confirm the reproducibility. At the turn-round humidity $(\mathrm{RH}=91-92 \%)$ from increasing to decreasing-humidity process, the samples were rested for $30 \mathrm{~min}$.

\section{Results}

\subsection{Water layer thicknesses under full hydration conditions measured with SAXS.}

The hydration state is known to be constant under full hydration conditions (molar ratios of $R=\left[\mathrm{H}_{2} \mathrm{O}\right] /[$ lipid] $>40)$, i.e., the thickness of the water layer $\left(d_{w}\right)$ between the membranes is saturated at a constant value. ${ }^{17)}$ This was confirmed for our prepared lipid samples $\left(R=\left[\mathrm{H}_{2} \mathrm{O}\right] /[\right.$ lipid $]=95,125$, and 155$)$ by subtracting the membrane thickness from the lamellar repeat distance measured by SAXS to obtain the water layer thickness (Fig. 1(b)). The constant water layer thickness indicates that the hydration states are the same for each concentration, but when the two lipids are compared, the water layer thicknesses are different: the PC lipid membranes are swollen $\left(d_{w}=25 \AA\right)$, while only a small number of water molecules is necessary to fully hydrate the PE membranes $\left(d_{w}=12 \AA\right)$. This thinner water layer thickness of PE lamellar phase than that of PC lamellar phase is consistent with the reported results. ${ }^{33)}$

\subsection{Hydration states of PC and PE lipids observed with THz-TDS.}

The THz spectra of the lipid dispersions are shown in Figs. 2a and 2(b). In this frequency range no signal arising from the lipid molecule itself appears; ${ }^{17)}$ that is, the spectra reflect only the dynamics of the water molecules. The THz spectrum of water in the $0.3-2.7 \mathrm{THz}$ range is known to include three dynamical modes: slow relaxation (with a relaxation time of $\tau=5 \mathrm{ps}$ ), fast relaxation ( $\tau=0.27 \mathrm{ps}$ ), and an intermolecular stretching vibration mode (at about $5 \mathrm{THz}){ }^{16,34)}$ The most dominant mode is the slow relaxation due to the reorientation dynamics, which is considered to be slowed further by the hydration effect of solutes, i.e. the 

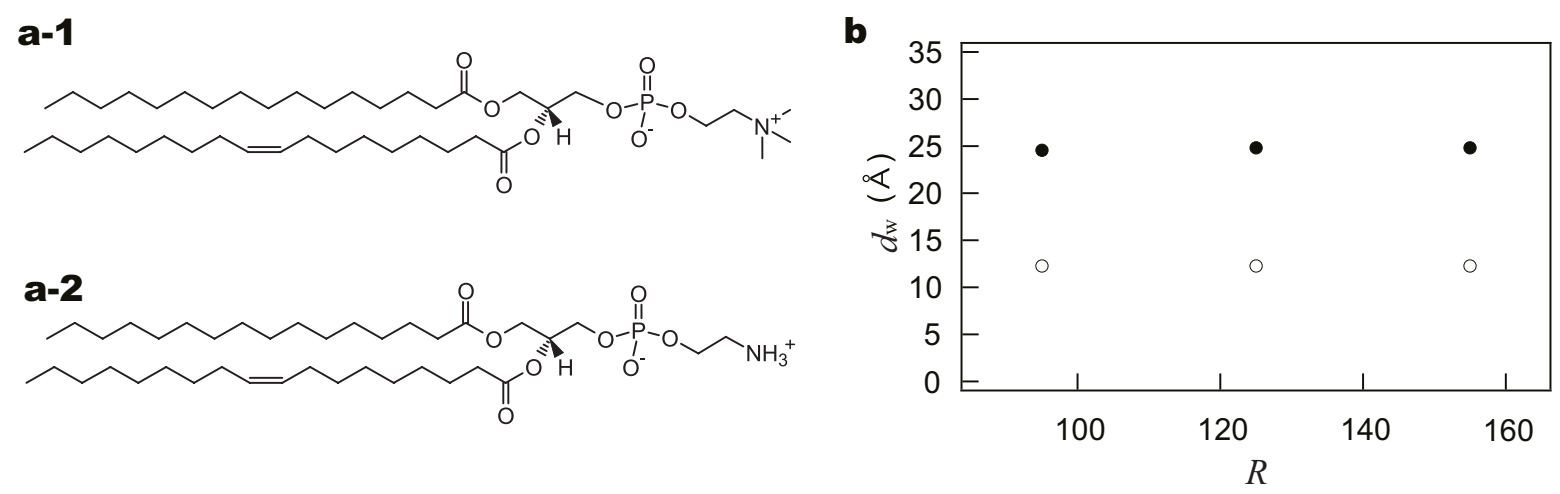

Fig. 1. Lipid structures and water layer thickness. (a) Molecular structures of (a-1) POPC (PC lipid) and (a2) POPE (PE lipid). (b) The thickness of the water layer between the lipid membranes at each concentration measured by SAXS. Closed circles are the results of the PC lipid, and open circles are those of the PE lipid.

water molecules are trapped by the solute, and this appears as a shift to lower frequencies in the GHz region. ${ }^{35)}$ The slowed dynamics, therefore, do not appear in the THz spectrum. $\mathrm{THz}$ spectroscopy is unable to distinguish waters belongings to the first and second hydration layers, but it provides information averaged over them. The dielectric constant in the $\mathrm{THz}$ region thus decreases in proportion to the amount of overall hydration water present, which is clearly observed in the imaginary part. ${ }^{19,36}$ ) The fast relaxation and stretching vibration modes are less affected by the hydration effect and are invariant between bulk and hydration water, ${ }^{34)}$ since these are transient dynamics. It has been suggested that the hydration effect on some proteins could accelerate the reorientation, ${ }^{37)}$ which should be reflected as an increase in the dielectric constant in the THz region. However, such an increase due to acceleration has not been reported in literature.

Since all samples of $R=95-155$ are in the full hydration condition where the water layer thicknesses are saturated and bulk water phases exist, the hydration states have to be the same for these samples. However, since the $\mathrm{THz}$ spectra include information of water dynamics not only in between lipid membranes but also in bulk phase, the change in the $\mathrm{THz}$ spectrum depending on $R$ is due to both an effect of volume fraction of water in the sample and an effect of hydration. With regard to the effect of volume fraction, the intensity of the spectrum is proportional to the volume fraction of water in the samples and the spectrum decreases as increasing the concentration of lipid.

As shown in Fig. 2a, the imaginary part of the PC lipid dielectric constant decreases as the concentration of the lipid increases, whereas the imaginary part of the PE dielectric constant (Fig. 2(b)) shows a smaller decrease with increasing concentration. A comparison of the 
spectra (Fig. 2(c)) at the same concentration $(R=125)$ more clearly shows the difference. This different behavior of decrease indicates that the overall long-range hydration states including the second hydration layer of the PC and PE lipids largely differ, since the volume fractions of lipids are not so different each other, i.e., the effects of volume fractions on the spectral change are similar. ${ }^{38)}$

To reduce the effect of volume fraction from the resultant spectrum, the normalized value of the imaginary part of the dielectric constant at $0.5 \mathrm{THz}$ (Fig. 2(d)) was found to offer a good indication of the hydration state. ${ }^{19)}$ Deviation of the normalized value from that of pure water results from the effect of hydration. Since amount of hydration water is proportional to the amount of lipids in the solution, the normalized value changes more largely from the result of pure water at the higher concentration of lipids. The results for the PC lipid show that the normalized value decreases, i.e., the water dynamics are retarded, which is consistent with previous work. ${ }^{17,19,34,36)}$ However, the increase in the normalized value of the PE lipid dispersion indicates acceleration of water dynamics when the dynamics is averaged for the first and second hydration layers of the PE lipid membrane.

In Fig. 2(e), the THz spectra of partially dried lipid films are shown. The samples were exposed in air with $65 \%$ of relative humidity. In this case, water evaporated almost and the spectra come from the dynamics of a small number of remaining water. In contrast to the results of $R=125$, the intensity of the spectrum of PE lipid film is smaller than that of PC lipid film, and it is almost zero. Considering above mentioned characteristics of the hydration water on each lipid membrane, it is suggested that most of water evaporated from the PE lipid film whereas some amount of bulklike water remain in the PC lipid film at this humidity. The inversion of the intensities of the spectra of PC and PE lipids between the results of $R=125$ and the lipid films suggests that the evaporation efficiencies of water from the lamellae of each lipid differ, presumably due to the different behavior of water between these lipid membranes.

Since the PC and PE lipids differ only by the ammonium group in the head group, the differences in the hydration states are considered to originate from the ammonium groups. The THz-TDS spectra for ammonium chloride and tetramethylammonium chloride (Fig. 3) shows that the dielectric constant of the ammonium chloride solution is larger than that of pure water, while that of tetramethylammonium chloride is smaller. The water dynamics are thus accelerated around the ammonium ions and slowed around the tetramethylammonium ions. Since an increase in the $\mathrm{THz}$ spectral signal was reported for $\mathrm{NaCl}$ solution, ${ }^{39)}$ it would appear that the acceleration is not specific to ammonium chloride. The hydration state of the ammonium group at the lipid head groups that directly face the water layer thus have a 
dominant effect on the water reorientation dynamics on the lipid membrane surfaces.

IR spectroscopy reveals the hydration state in the first hydration shell, since only $\mathrm{OH}$ band is observed. Previous IR spectroscopy results showed that the $\mathrm{OH}$ band $v_{13}\left(\mathrm{H}_{2} \mathrm{O}\right)$ at around 3400 and $3250 \mathrm{~cm}^{-1}$ shifts to a higher frequency $\left(3600 \mathrm{~cm}^{-1}\right)$ due to the hydration effect for both hydration shells of PC and PE lipids. ${ }^{40)}$ Since this shift to higher frequency is due to the restriction of water dynamics, ${ }^{41)}$ the water dynamics in the first hydration shells of the PC and PE lipids are both restricted. Our results, however, indicate that the reorientation dynamics at the membrane surfaces differ in the long-range hydration layer in which the second hydration layer is included. At the PC lipid membrane surface, the dynamics in the first and second hydration layers are mostly slowed, which is consistent with our previous work on a DMPC (1,2-dimyristoyl-sn-glycero-3-phosphocholine) system. ${ }^{17)}$ The long-range hydration state of POPC extends to $1 \mathrm{~nm}$ from the surface, as confirmed by the same analysis. For the PE membrane, the THz spectrum indicates the water dynamics in the long-range second hydration layer are accelerated, since averaged dynamics of the first and second hydration layer is accelerated while that in the first hydration shell is restricted. Previous assumptions for determining the hydration number from the THz-TDS signal cannot be applied in this case, and the precise thickness of the hydration layer is therefore unclear. However, we can expect the thickness have similar length scale to that of the PC lipid, since the increase in the PE lipid dielectric constant at $0.5 \mathrm{THz}$ is roughly the same as the decrease for the PC lipid (Fig. 2(d)). This water with accelerated dynamics is likely to be equivalent to the 'hypermobile water' reported at the surface of actin molecules. ${ }^{37)}$

To estimate a relaxation time of the accelerated reorientation dynamics of water at the surface of PE lipid, fitting calculations to the data were performed. For the fitting, Debye functions for each relaxation modes are superposed as a first-step approximation, ${ }^{16)}$ although such a superposition of Debye functions is naive for detailed estimation. ${ }^{34)}$ Besides the slow and fast relaxation modes and the intermolecular stretching vibration mode, a third relaxation mode is also added for the accelerated slow relaxation mode (accelerated hydration water), and the total dielectric constant at each frequency $\epsilon(v)$ is:

$$
\epsilon(v)=\frac{S_{s}}{1+i\left(2 \pi \nu \tau_{s}\right)}+\frac{S_{f}}{1+i\left(2 \pi v \tau_{f}\right)}+\frac{S_{a}}{1+i\left(2 \pi \nu \tau_{a}\right)}+\frac{A_{v}}{v_{v}^{2}-v^{2}+i v\left(\gamma_{v} / 2 \pi\right)}+\epsilon_{\infty}
$$

where, the first and second terms are the slow and fast relaxation modes respectively, and the third term is due to the accelerated slow relaxation mode. Final term is from the intermolecular stretching vibration mode. The relaxation times of the slow and fast relaxation $\left(\tau_{s}\right.$ and $\left.\tau_{f}\right)$, the strength $S_{f}$ of the fast relaxation, and the frequency and damping constant in the inter- 
molecular stretching vibration mode $\left(v_{v}, \gamma_{v}\right)$ were determined from the fitting for pure water with fixing $S_{a}=0$. These parameters were fixed, and then, the measured dielectric constants of PE lipid samples are fitted, in which $\tau_{a}$ was made to be constant value for each concentration samples. The fitted lines are depicted in Fig. 2 (b), and as a result, the relaxation time of the accelerated slow relaxation mode is obtained as $\tau_{a}=1.5 \mathrm{ps}$. This indicates the accelerated hydration water have about three times faster reorientation dynamics compared to bulk water $\left(\tau_{s}=5 \mathrm{ps}\right)$. Tendency of the acceleration is consistent with the hypermobile water $(\tau=1 / 2 \pi v$ $=26 \mathrm{ps}$ is accelerated to be $4 \mathrm{ps}$ ), although the absolute values cannot be compared since temperatures are different. ${ }^{37)}$ Further, from the fitting results of $S_{s}$ and $S_{a}$, it is confirmed that the hydration layer on PE membranes also ranges long as observed for PC membranes (several layers of water molecules). Besides, it is also indicated that the accelerated water make up the majority in the long-range hydration layer of PE membranes. For the PC lipid, the measured dielectric constants were fitted well as shown in Fig. 2 (a), even when $S_{a}$ was fixed to 0 . This confirms that the relaxation time of the hydration water is over $5 \mathrm{ps}$ and the retarded relaxation mode is not observed in $\mathrm{THz}$ region. This is consistent with reported results about dynamics of hydration water around PC membranes ${ }^{17,20)}$ or other solutes. ${ }^{19,34,36)}$

\subsection{Humidity dependent structural transitions of PC and PE membranes observed with GISAXS.}

The comparable length scales of the hydration layer thickness and membrane interaction distance suggest a correlation between the hydration state and the structural changes of the lipid membrane, in a similar manner to that reported in our study of a nonionic surfactant system. ${ }^{19)}$ The structural changes during the lamellar to inverted hexagonal phase transition were observed using GISAXS in a humidity-controlled atmosphere. ${ }^{24)}$ Partially dried lipid films on a substrate were created by stacking a number of lipid membranes, and the hydration state can be controlled through the humidity; dehumidification results in water molecules detaching from the lipid film. As the amount of water between the membranes decreases, the membranes come into close contact and begin to undergo the phase transition.

The GISAXS profiles of the PC and PE lipid films under different humidity conditions (Fig. 4) show that not only the PE lipid ${ }^{24)}$ but also the PC lipid exhibits a phase transition from a lamellar to inverted hexagonal phase during dehumidification. The GISAXS patterns of POPC and POPE at phase transition points are compared in Fig. 5 with indexes. From this indexes, it is confirmed that an inverted hexagonal phase which has space group $\mathrm{p}^{27)}$ is emerged from a lamellar phase. In is noted that no other phase, such as a rhombohedral 
phase as reported for other lipids, ${ }^{24,25,27,28)}$ was observed between the lamellar and inverted hexagonal phases. Since the head group of PC lipid has larger volume than that of PE lipid and the hydrophobic acyl chains are completely the same, the molecular shapes are much different (PE membrane has certainly negative but PC membrane has nearly zero curvature). Thus, the results imply that the transition is not determined only by the molecular shapes of the lipids. We note that the phase transitions occur at different humidities (PC lipid: $\mathrm{RH}=40-51 \%$; PE lipid: RH $=86-90.5 \%$ ). We also note that the lamellar structures are similar (Fig. 5) as similar scattering patterns are observed at each phase transition point. At the transition points, the lamellar repeat distances are calculated to be 45.0 and $46.8 \AA$ for the POPC and POPE lipids, respectively. At constant humidity, the lamellar repeat distance of the PC lipid is longer than that of the PE lipid (51.8 $\AA$ for PC and $47.8 \AA$ for PE at $90.5 \%$ ), which is consistent with our THz results on the partially dried lipid films (Fig. 2(e)) and SAXS results under full hydration conditions (Fig. 1(b)). Since water is easily ejected from the space between the PE membranes, the PE lipid film exhibits a ribbon $\mathrm{P}_{\alpha}$ phase, ${ }^{42)}$ i.e., the collapsed inverted hexagonal phase, under low humidity conditions.

We can thus conclude that the PC lipid membranes are swollen more easily than the PE membranes. At a constant humidity, the chemical potential of water molecules in the lamellar phases are equilibrated to be the same as that of vapor in the atmosphere, and the chemical potential of water in the vapor is determined by the relative humidity. The results that the same lamellar repeat distance is achieved at lower humidity condition for PC lipid membranes indicates the chemical potential of water between the PC lipid membranes is smaller when the potentials are compared for the same lamellar repeat distance. Furthermore, the GISAXS results imply that the structural transition is determined by the repeat distance of lamellae, i.e., water content in the lamellae, in addition to the molecular shape of the lipid, which are independent variables. The transition occurs when lipid membranes come close each other, even when the membrane does not have negative curvature. It is easier on the PE lipid membranes to achieve the thin water layer necessary for the transition through dehydration due to the larger chemical potential of the hydration water.

\section{Discussion}

Here, we concisely summarize the experimental results: (1) Water layer thickness between PC lipid membranes is much larger than that of PE lipid in full hydration condition. (2) Although the first hydration shells are almost the same for PC and PE lipids, the second hydration layers differ largely: While water is slowed down between PC membranes, it is 
mostly accelerated between PE ones. (3) The structural transition from lamellar to inverted hexagonal phases is dominated by the water content in the lamellae in addition to the molecular shape of lipids. (4) The lamellar repeat distances in lipid films are determined by the chemical potentials of water between membranes for both lipids. (5) The chemical potential of water is smaller between PC membranes than between PE membranes if compared at the same structure of the lipid membranes.

Because water behaves differently in the hydration layers of the PC and PE lipids, it is natural that the chemical potentials of water also differ at the same repeat distance, since the chemical potential of hydration water is determined by the hydrogen-bond network in the water phase and its entropy, which are dependent on the interactions between lipids and water. Thus, these five facts from the independent three experiments are all consistent. That is, in summary, the hydration state affects the lamellar repeat distance, which determines the structural transition of the membrane, i.e., the lamellar to inverted hexagonal phase transition is dominated by the water behavior in the long-range hydration layer. Actually, the effects of the water behavior and of the membrane curvature seem to work cooperatively. The slight difference of the lamellar repeat distances of PC and PE lipids at each phase transition point (Fig. 5) seems to come from the effect of curvature of membrane. It is possible that the hydration state also influence on the curvature of the membrane, since hydration water is possibly regarded as a part of head group.

The lamellar repeat distance of lipid membranes in the full hydration condition has been interpreted by direct interactions between membranes: The balance of the attractive (van der Waals interaction) and the repulsive (steric repulsion due to membrane fluctuations called Helfrich repulsion ${ }^{43,44)}$ and short-range repulsion) interactions determines the membranemembrane distance. ${ }^{18)}$ The theory typically assumes a priori that the hydration effect of the lipid membrane is little, since only the first hydration shell has been observed in past studies. $\left.{ }^{6}\right)$ Since PE membrane is stiffer about $20 \%$ than the PC membrane, ${ }^{45)}$ the Helfrich repulsive force is smaller for PE membranes. Therefore, the thinner water layer thickness in the fully hydrated PE membranes than that in PC membranes is interpreted partially by the different Helfrich repulsion between them. On the other hand, the difference in the water layer thicknesses seems to be too large to be interpreted only by the different repulsive forces. Further, our results indicate the distance in the partially dried lipid film is dominated by water chemical potential. The relation between these mechanisms for determining the lamellar repeat distances in partially dried and fully hydrated samples is still not clear. The different water behaviors in the long-range hydration layers from bulk water possibly influence the interac- 
tions, since for example it is possible that the dielectric constant and the viscosity of hydration water differ from those of bulk water, which are related to the interactions. Therefore, the relation between the long-range hydration layer and the interaction between lipid membranes should be investigated more in the future.

The different long-range hydration states between PC and PE lipids are expected to originate from the fractionation between highly ordered and less-ordered regions of the water molecular arrangement around a hydrophilic solute, as has recently been reported. ${ }^{46)}$ As the head group of the PC lipid is less hydrophilic than that of the PE lipid since three methyl groups face water phase on PC head group, our THz-TDS results are consistent with the assumption that the hydration water around the PE head group is fractionated: The water in the less-ordered region is released from the hydrogen-bond network and thus has faster reorientation dynamics than bulk water. The head group of the PC lipid is less hydrophilic, and a less-ordered region is not expected to emerge.

Different hydration water behavior depending on the solute hydrophilicity is clearly reflected in our THz-TDS results of ammonium chloride and tetramethylammonium chloride. While the dynamics of the less hydrophilic hydration water on tetramethylammonium chloride are slowed in most of the hydration layers, the better hydrophilic hydration water dynamics on ammonium chloride are accelerated. This means that the fractionation of the hydration water is generally related to the hydrophilic or hydrophobic (less hydrophilic) hydration. In this respect, THz-TDS has proven to be a very powerful tool since this fractionation can be distinctly identified in the long-range hydration layer of the solute including a second hydration layer.

The present results indicate that the self-assembly of biomolecules and soft materials is affected by the behavior of the surrounding water. Water is not simply a homogeneous background, and it is highly possible that living systems exploit the specific behavior of water for survival. This cooperativity is also important when designing industrial materials dispersed in water. It may be possible to control meso- and macroscopic properties of lipids, surfactants, polymers, gels, and colloids by the microscopic chemical design of particular molecules considering their hydration states. The role of water and, specifically, the behavior of water for self-assembly requires further investigation.

\section{Conclusion}

Considering through the whole results of SAXS, THz-TDS and GISAXS, several conclusions and issues are raised. First of all, the phase transition from the lamellar to inverted 
hexagonal phases is not determined only by the molecular shapes of lipids. Further, it is concluded that the structural phase transition from lamellar to inverted hexagonal phases, the intermediated process of membrane fusion, is affected by the different hydration states between PC and PE lipids. That is, both independent variables, molecular shapes of lipids and the water content in the lamellae, are found to determine the phase transition. Since water molecules are more easily detached from PE lipid membranes, the PE membranes readily approach each other and easily undergo the structural transition. Although the effects of hydration water have long been ignored, our study indicates that the behavior of the hydration water needs to be considered in understanding the structural changes of the lipid membrane and is universally important in the self-assembly mechanisms exhibited by various biomolecules and soft materials dissolved in water. Furthermore, to understand the cooperativity between water and lipid for the self-assembly, the need for a new theory which takes into account the long-range hydration state is raised beyond the balance of the direct interactions. In addition to the physical and biological aspects, it is revealed that the difference in the hydration states is based on the chemistry of hydrophilic and hydrophobic hydration. A less hydrophilic hydration layer will slow the water dynamics, while a better hydrophilic hydration layer accelerates the dynamics.

\section{Acknowledgments}

We thank Dr. N. L. Yamada (High Energy Accelerator Research Organization, Japan) and Dr. U-Ser Jeng (National Synchrotron Radiation Research Center, Taiwan) for their assistance with the GISAXS experiments (Proposal No. 2011-1-150-5). M.H. is supported by a Grantin-Aid for Scientific Research from JSPS (Grant No. 42740289) and the Murata Science Foundation (H23-54). The SAXS experiments were performed under the approval of the Photon Factory Program Advisory Committee (Proposal Nos. 2011G516 and 2011G550). 

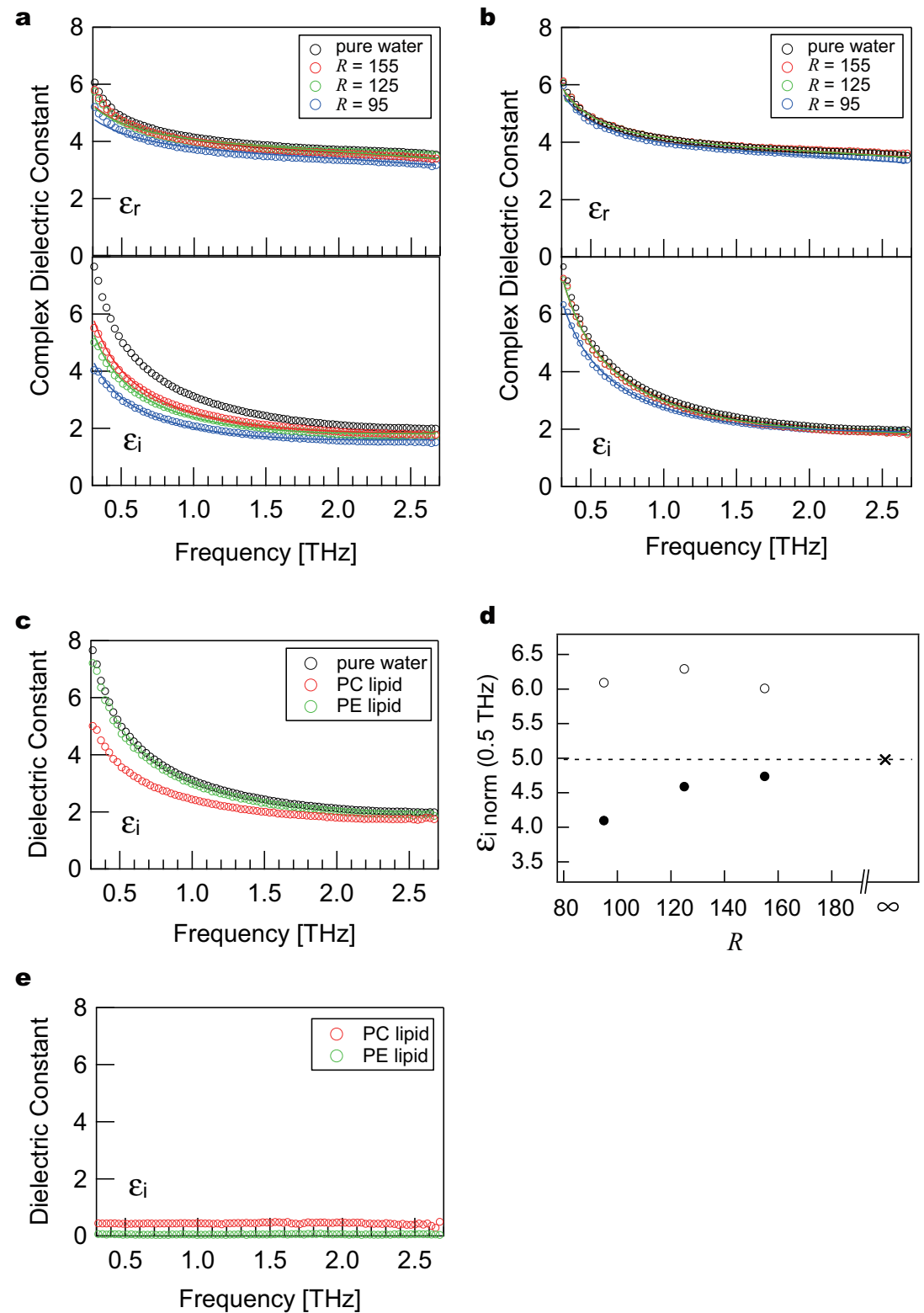

Fig. 2. Dielectric functions of the $\mathrm{PC}$ and $\mathrm{PE}$ lipids in $\mathrm{THz}$ region. Complex dielectric constants of the (a) $\mathrm{PC}$ and (b) PE lipid dispersions in the THz frequency range at $50{ }^{\circ} \mathrm{C}$. The solid lines are the fitting results with eq. 1. The real and imaginary parts are shown in the upper and lower plots, respectively. (c) Comparison of the imaginary part of the dielectric constant of the PC and PE lipids at the same concentration $(R=125)$ and that of pure water. (d) Normalized values of the imaginary part of the dielectric constant at $0.5 \mathrm{THz}$ for the PC (closed circles) and PE (open circles) lipids. The cross mark is the result of pure water $(R=\infty)$; the dashed line is a guide. (e) Comparison of the imaginary part of the dielectric constants of partially dried lipid films at about RH $=65 \%$. 


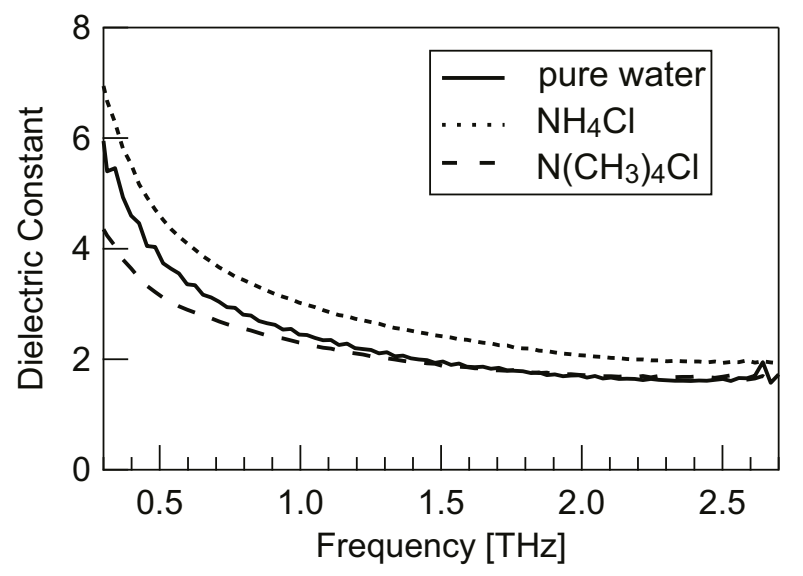

Fig. 3. Imaginary dielectric constant of the ammonium groups. Imaginary part of the dielectric constant in the $\mathrm{THz}$ region for $2 \mathrm{M}$ aqueous solutions of ammonium chloride $\left(\mathrm{NH}_{4} \mathrm{Cl}\right)$ and tetramethylammonium chloride $\left(\mathrm{N}\left(\mathrm{CH}_{3}\right)_{4} \mathrm{Cl}\right)$ at $25^{\circ} \mathrm{C}$.
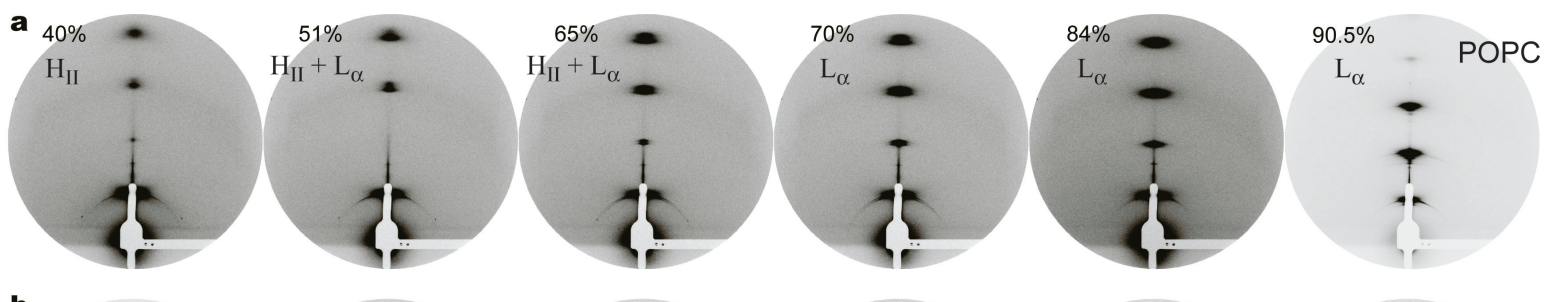

b $39 \%$
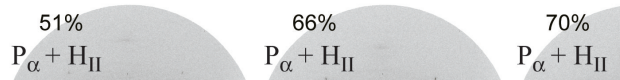

$86 \%$
$\mathrm{H}_{\text {II }}$

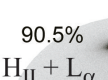

POPE
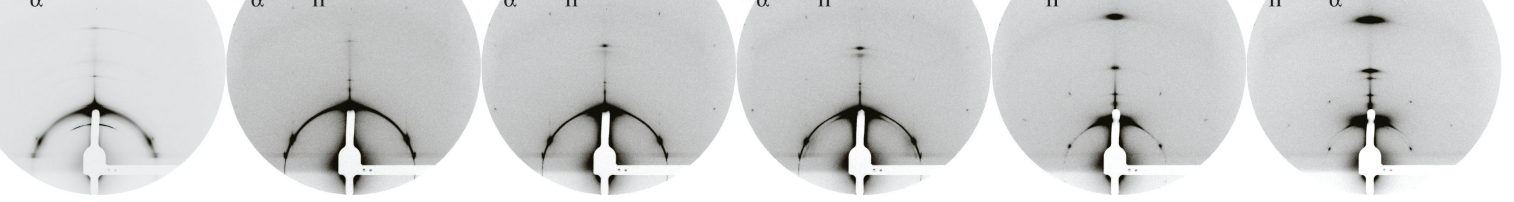

Fig. 4. Grazing-incidence small-angle X-ray scattering profiles of the POPC and POPE lipid films. Profiles of the (a) POPC and (b) POPE lipid films under different humidities conditions at $50{ }^{\circ} \mathrm{C}$. The relative humidity and phase of the lipid are shown in the upper left of each profile $\left(\mathrm{L}_{\alpha}\right.$ : lamellar phase; $\mathrm{H}_{\mathrm{II}}$ : inverted hexagonal phase; $\mathrm{P}_{\alpha}$ : ribbon phase). Specular reflection due to the layer stacking is observed along the central line, while off-specular reflection spots due to lateral ordering in the inverted hexagonal and ribbon phases are observed off the central line. 


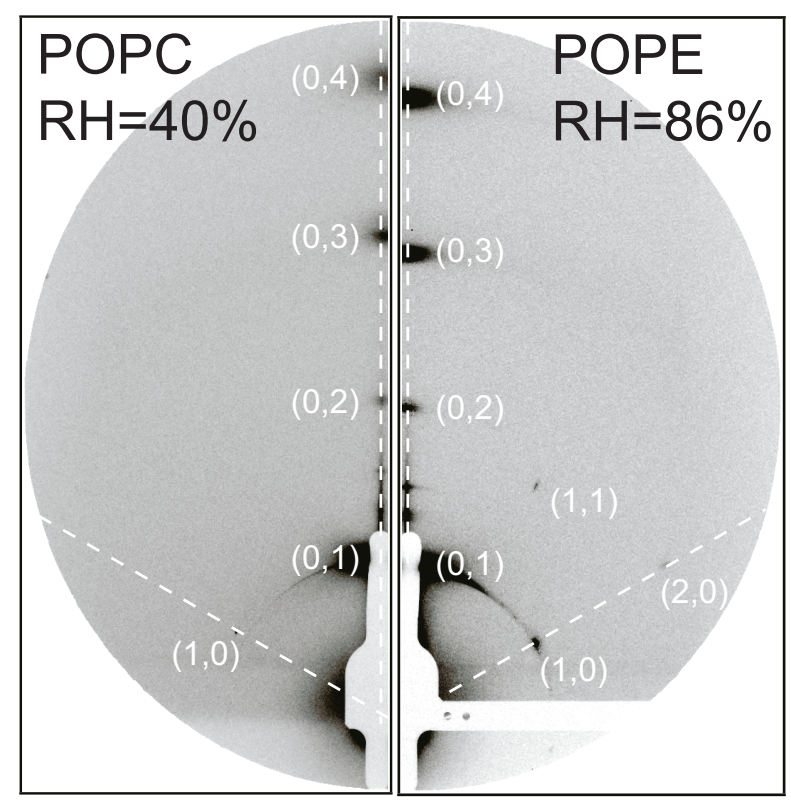

Fig. 5. Grazing-incidence small-angle X-ray scattering profiles at the phase transition points. Profiles of the POPC (left; $\mathrm{RH}=40 \%$ ) and POPE (right; $\mathrm{RH}=86 \%$ ) at the transition from the inverted hexagonal to lamellar phase. Each diffraction peak is indexed $\left(q_{x}, q_{z}\right)$. The vertical dashed line is directed to $q_{z}$, and the angle between the vertical line and each side dashed line is $60^{\circ}$. 


\section{References}

1) M. Chaplin: Nat. Rev. Mol. Cell Biol. 7 (2006) 861.

2) J. Israelachvili, H. Wennerström: Nature 379 (1996) 219.

3) B. V. Derjaguin, L. Lau: Acta Phys. Chim. URSS 14 (1941) 633.

4) E. J. W. Verwey, J. Th. G. Overbeek: Theory of the stability of lyophobic colloids, (Dover Publications, New York, 1999).

5) J. N. Israelachvili: Intermolecular surface forces (Academic Press, Elsevier, Amsterdam, 2010).

6) J. Milhaud: Biochim. Biophys. Acta 1663 (2004) 19.

7) S. König, E. Sackmann, D. Richter, R. Zorn, C. Carlile, T. M. Bayerl: J. Chem. Phys. 100 (1994) 3307.

8) J. Swenson, F. Kargl, P. Berntsen, C. Svanberg: J. Chem. Phys. 129(2008) 045101.

9) R. Buchner, J. Barthel, J. Stauber: Chem. Phys. Lett. 306 (1999) 57.

10) B. Ratajska-Gadomska, B. Białkowski, W. Gadomski, Cz. Radzewicz: Chem. Phys. Lett. 429 (2006) 575.

11) S. Ebbinghaus, S. J. Kim, M. Heyden, X. Yu, U. Heugen, M. Gruebele, D. M. Leitner, M. Havenith: Proc. Natl. Acad. Sci. USA. 104 (2007) 20749.

12) M. Ahmad, W. Gu, T. Geyer, V. Helms: Nat. Commun. 2 (2011) 261.

13) S. H. Chong, S. Ham: Proc. Natl. Acad. Sci. USA. 109 (2012) 7636.

14) B. Ferguson, X.-C. Zhang: Nat. Mater. 1 (2002) 26.

15) C. Rønne, P. O. Åstrand, S. R. Keiding: Phys. Rev. Lett. 82 (1999) 2888.

16) H. Yada, M. Nagai, K. Tanaka: Chem. Phys. Lett. 464 (2008) 166.

17) M. Hishida, K. Tanaka: Phys. Rev. Lett. 106 (2011) 158102.

18) H. I. Petrache, N. Gouliaev, S. Tristram-Nagle, R. Zhang, R. M. Suter, J. F. Nagle: Phys. Rev. E. 57 (1998) 7014.

19) M. Hishida, K. Tanaka: J. Phys. Condens. Matter 24 (2012) 284113.

20) D. H. Choi, H. Son, S. Jung, J. Park, W. Park, O. S. Kwon, G. Park: J. Chem. Phys. 137 (2012) 175101.

21) L. V. Chernomordi, M. M. Kozlov: Nat. Struct. Mol. Biol. 15 (2008) 675. 
22) K. Emoto, T. Kobayashi, A. Yamaji, H. Aizawa, I. Yahara, K. Inoue, M. Umeda: Proc. Natl. Acad. Sci. USA. 93 (1996) 12867.

23) J. N. Israelachvili, D. J. Mitchell, B. W. J. Ninham: Chem. Soc. Faraday Trans. 72 (1976) 1525.

24) L. Yang, H. W. Huang: Science 297 (2002) 1877.

25) L. Yang, L. Ding, H. W. Huang: Bochemistry, 42 (2003) 6631.

26) A. Tardieu, V. Luzzati: J. Mol. Biol., 75 (1973) 711.

27) L. Ding, W. Liu, W. Wang, C. J. Glinka, D. L. Worcester, L. Yang, H. W. Huang: Langmuir, 20 (2004) 9262.

28) L. Yang, H. W. Huang: Biophys. J., 84 (2003) 1808.

29) P. M. Kasson, E. Lindahl, V. S. Pe: J. Am. Chem. Soc. 133 (2011) 3812.

30) M. Nagai, H. Yada, T. Arikawa, K. Tanaka: Int. J. Infrared Millimeter Waves 27 (2006) 505.

31) N. Kučerka, M. Nieh, J. Katsaras: Biochim. Biophys. Acta 1808 (2011) 2761.

32) M. Rappolt, A. Hickel, F. Bringezu, K. Lohner: Biophys. J. 84 (2003) 3111.

33) R. P. Rand, N. Fuller, V. A. Rersegian, D. C. Rau: Biochemistry 27 (1988) 7711.

34) T. Arikawa, M. Nagai, K. Tanaka: Chem. Phys. Lett. 457 (2008) 12.

35) B. Klösgen, C. Reichle, S. Kohlsmann, K. D. Kramer: Biophys. J. 71 (1996) 3251.

36) U. Heugen, G. Schwaab, E. Bründermann, M. Heyden, X. Yu, D. M. Leitner, M. Havenith: Proc. Natl. Acad. Sci. USA. 103 (2006) 12301.

37) S. R. Kabir, K. Yokoyama, K. Mihashi, T. Kodama, M. Suzuki: Biophys. J. 85 (2003) 3154.

38) R. S. Armen, O. D. Uitto, S. E. Feller: Biophys. J. 75 (1998) 734.

39) P. U. Jepsen, H. Merbold: J. Infrared Milli. Terahz. Waves 31 (2010) 430.

40) D. R. Gauger, H. Binder, A. Vogel, C. Selle, W. Pohle: J. Mol. Struct. 614 (2002) 211.

41) H. Binder: Eur. Biophys. J. 36 (2007) 265.

42) B. Kent, C. J. Garvey, D. Cookson, G. Bryant: Chem. Phys. Lipid. 157 (2009) 56.

43) W. Helfrich: Z. Naturforsch. 33a (1978) 305.

44) R. Podgomikt, V. A. Parsegian, Langmuir 8557 (1992).

45) E. Evans, D. Needham: J. Phys. Chem. 91 (1987) 4219.

46) A. Godec, J. C. Smith, F. Merzel: Phys. Rev. Lett. 107 (2011) 267801. 